\title{
Gain-controlled wave chaos in a chaotic optical fibre
}

\author{
C. Michel \\ LPMC - Université de Nice, Nice, France \\ claire.michel@unice.fr \\ S. Tascu \\ LPMC - Université de Nice, Nice, France \\ sorin.tascu@unice.fr \\ V. Doya \\ LPMC - Université de Nice, Nice, France \\ O. Legrand \\ olivier.legrand@unice.fr \\ LPMC - Université de Nice, Nice, France \\ F. Mortessagne \\ fabrice.mortessagne@unice.fr \\ LPMC - Université de Nice, Nice, France
}

In this paper, we present a non-standard fibre amplifier specially designed to amplify scar modes of a multimode chaotic optical fibre. More precisely, we introduce Ytterbium in the optical fibre as a gain medium localised on the maximum of intensity of the scar modes. After briefly recalling the relevance of a chaotic optical fibre as a device to visualise quantum chaos, we describe the amplification process of scars. We present some numerical results that demonstrate the selective amplification of scar modes, with an amplification rate proportional to the overlap between these modes and the gain area. [DOI: 10.2971/jeos.2009.09020]

Keywords: wave chaos, scar modes, chaotic optical fibre, double-clad Ytterbium-doped fibre amplifiers, localised gain

\section{INTRODUCTION}

For now a few years, a lot of applications in optics have taken advantage of wave chaos. The interest is principally based on some essential properties of the intensity distribution of the modes in two dimensional chaotic cavities [1]. The intensity of generic modes is statistically uniformly distributed over the cavity. For some applications described below, this uniformity constitutes an important advantage. However, some modes, called scars, don't follow this generic behaviour. They localise the maximum of their intensity along particular directions of the cavity. These properties may also have some applications, as shown in the following.

With the constant need of higher compactness of components for integrated photonic circuits, microcavity lasers have known, for the past few years, active development [2]. These investigations are not only guided by applications, but also by the perspective of fundamental physics [3, 4]. Different materials can be used as microcavities. For instance, one can find polymer microlasers [5] that allow electrical injection, lithium niobate resonators that highlight photorefractive properties [6], and many others. One of the common goals of those investigations is to improve the efficiency of the lasing modes, as well as to control their directionality, or their quality factor. To meet the latter requirements, the geometry of the microcavities has been modified, in order to make them chaotic $[7,8]$. The lasing modes of such cavities are mostly whispering gallery modes that have generally the lowest lasing threshold [3]. Modes that build on periodic orbits of the cavity (scars) are also extremely attractive. These specific modes have the best directionality [9].

Wave chaos also may suggest very interesting solutions, in guided optics. Indeed, many applications in optical communication require the use of double clad fibres (DCF) [10]. DCF used as amplifiers ensure a high coupling between a high power multimode pump, propagating in a large cross section inner cladding, and a single-mode signal, propagating in a rare-earth doped core. The important parameter in DCF amplifiers is the overlap integral between the pump field intensity distribution and the doped area. Each mode is considered to be absorbed proportionally to the overlap of the pump field with the gain medium. It has been shown [11] that the more uniform the distribution of intensity is, the more the absorption is effective. It is well-known that the modes intensity distribution strongly depends on the shape of the inner cladding. The use of a chaotic geometry for the inner cladding [12, 13] allows to achieve an optimised absorption, since the generic modes of such a chaotic cladding have an uniform spatial distribution of intensity. Thus, in integrated optics as well as in guided optics, wave chaos has proved to be a very powerful tool. Integrated optics takes advantage of very specific and non standard modes of a chaotic cavity, whereas guided optics takes advantage of the (speckle) modes that follow the generic behaviour of such cavities.

In previous works, we showed that multimode optical fibres are particularly well adapted for fundamental investigations 
in the domain of wave chaos [12], in particular to obtain and analyse scar modes in an optical fibre [14]. In this paper, we claim to go towards the control of wave chaos manifestations by the introduction of gain in the optical fibre. As a selective excitation is practically not feasible, we present an original way to achieve a selective amplification of scar modes, inspired by the fact that our device is perfectly adapted to optical amplification. More precisely, we propose to enhance scar modes by positioning a gain medium, all along the fibre, in the vicinity of the maximum of intensity of a family of scars. In this paper, we present some numerical results, based on realistic parameters, that demonstrate both how scar modes can be enhanced, and the feasibility of the experiment.

\section{MODEL FOR THE WAVE PROPAGATION IN A CHAOTIC OPTICAL FIBRE}

In this section, we consider a passive highly multimode step-index fibre, with respective indices of the core and the cladding, $n_{c o}=1.451$ and $n_{c l}=1.41$. The transverse cross section of the fibre is a disk of radius $R$ truncated at a distance $R_{c}=R / 2$ from the centre, as shown in Figure 1.

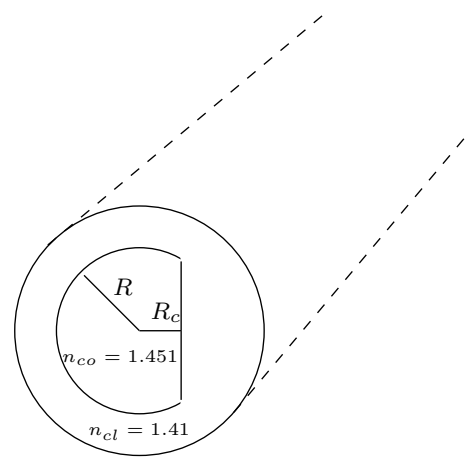

FIG. 1 Sketch of the transverse cross section of the D-shaped multimode fibre studied in this paper.

\subsection{Modal decomposition}

\subsubsection{Analogy between Helmholtz and Schrödinger equations}

We denote by $z$ the position along the axis of the fibre, and by $\mathbf{r}$ the position in the transverse plane. The optical index is invariant along $z: n(\mathbf{r}, z)=n(\mathbf{r})$. Weak guidance legitimates the scalar approximation [15]. Thus, the electromagnetic field $\psi_{s}(\mathbf{r}, z)$ propagating in the truncated fibre obeys the scalar three-dimensional Helmholtz stationary equation

$$
\left(\Delta_{\perp}+\partial_{z z}\right) \psi_{s}(\mathbf{r}, z)+n^{2}(\mathbf{r}) k_{0}^{2} \psi_{s}(\mathbf{r}, z)=0
$$

where $\Delta_{\perp}$ is the transverse Laplacian and $k_{0}=2 \pi / \lambda_{s}$ is the vacuum signal wave number. Considering the expression of the field $\psi_{s}(\mathbf{r}, z)$

$$
\psi_{s}(\mathbf{r}, z)=\int d \beta \phi(\mathbf{r} ; \beta) e^{i \beta z}
$$

Eq. (1) can be reduced to

$$
\Delta_{\perp} \phi(\mathbf{r} ; \beta)+\left[n^{2}(\mathbf{r}) k_{0}^{2}-\beta^{2}\right] \phi(\mathbf{r} ; \beta)=0
$$

Let $n_{c l}^{2} k_{0}^{2}=\beta_{c l}^{2}$ and $n_{c o}^{2} k_{0}^{2}=\beta_{c o}^{2}$. For $\beta_{c l}^{2} \leq \beta^{2} \leq \beta_{c o}^{2}$, Eq. (3) is solved at discrete values $\beta_{n}$, called the propagation constants of the guided modes. Eq. (3) can be rewritten as an eigenvalue equation

$$
\left[-\frac{1}{2} \Delta_{\perp}+\frac{\beta_{c o}^{2}-n^{2}(\mathbf{r}) k_{0}^{2}}{2}\right] \phi(\mathbf{r} ; \beta)=\frac{\beta_{c o}^{2}-\beta^{2}}{2} \phi(\mathbf{r} ; \beta)
$$

that gives

$$
\left[-\frac{1}{2} \Delta_{\perp}+V(\mathbf{r})\right] \phi=E \phi
$$

with $V(\mathbf{r})=\frac{\beta_{c o}^{2}-n^{2}(\mathbf{r}) k_{0}^{2}}{2}$ and $E$, the eigenenergy, which takes on discrete values $E_{n}$, related to the $\beta_{n}$ 's through $\beta_{n}^{2}=\beta_{c o}^{2}-$ $2 E_{n}$. Eqs. (3) and (5) are formally equivalent, and show the analogy between the three-dimensional Helmholtz stationary equation and the stationary Schrödinger equation.

The average number of modes $N(\beta)$ up to a given value of $\beta$ in such a fibre is approximatively given by [12]

$$
N(\beta)=\frac{S}{4 \pi}\left(\beta_{c o}^{2}-\beta^{2}\right)
$$

For a given polarisation and for $\lambda_{s}=1064 \mathrm{~nm}$, the total number of modes is roughly given by $N\left(\beta_{c l}\right)(\simeq 3500)$.

\subsubsection{Paraxial approximation}

In the paraxial approximation, valid for the first hundreds guided modes [12], one can derive the Schrödinger equation in fictitious time $z$ :

$$
i \beta_{c o} \partial_{z} \varphi(\mathbf{r}, z)=\left[-\frac{1}{2} \Delta_{\perp}+V(\mathbf{r})\right] \varphi(\mathbf{r}, z)
$$

The chaotic optical fibre then allows a direct visualisation of a quantum wave evolution.

\subsection{Modes of a chaotic optical fibre}

In this section, we recall some of the principal properties of the modes in a chaotic cavity. This will constitute an introduction for the following parts.

\subsubsection{From WKB approximation to periodic orbits theory}

In the semiclassical approach, the most commonly used approximation is the WKB (Wentzel-Kramers-Brillouin) approximation. WKB approximation consists in writing the wave function as an exponential function in which the amplitude and the phase are slowly varying. The integrals involved in the calculation of the phase are easily performed when the motion can be describe in terms of action-angle variables. This description is fully valid for integrable systems. In such systems, there are at least as many constants of motion as degrees of freedom. For instance, in the two-dimensional circular billiard, the two constants of motion are the energy and the angular momentum with respect to the centre of the circle. Figure 2(a) presents a typical trajectory for which the motion is confined between inner and outer radii. The outer radius is the circular boundary of the billiard, and the inner radius is defined as a caustic, that surrounds a non visited region. As soon as there are fewer constants of motion than degrees of 


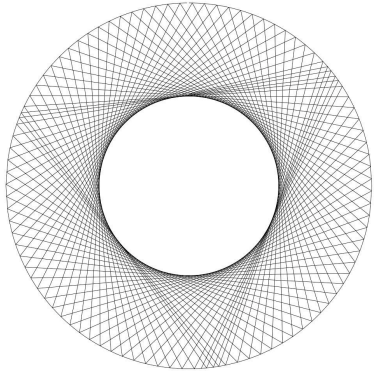

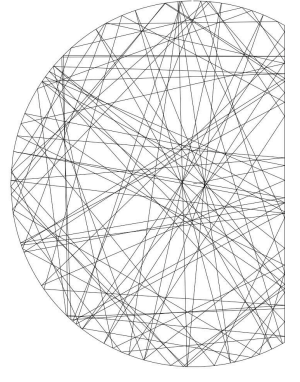

FIG. 2 Two examples of a single ray trajectory in 2-dimensional billiards : (a) in a circular billiard, (b) in a chaotic D-shaped billiard

freedom, the system is no more integrable, and the WKB approximation fails [16]. This is the case for chaotic billiards, in which the energy is the only constant of motion. In such systems, rays' dynamics is obviously drastically different. This is exemplified in Figure 2(b) for a truncated circle hereafter called the D-shaped billiard. This geometry induces chaotic dynamics, and to understand the waves behaviour in such a billiard, an other approach has to be used.

\subsubsection{Generic modes of a chaotic billiard : speckle (ergodic) modes}

In 1971, Gutzwiller introduced an entirely new approach, based on the knowledge of the unstable periodic orbits (PO) of the system [17]. His main result is the famous trace formula that exhibits a strong correspondence between the position of the resonances in the spectrum of a chaotic cavity and the POs. POs are trajectories that come back to their initial condition after a number $n$ of bounces on the boundary of the billiard. In a chaotic billiard the number of POs grows exponentially with their length [18] and each PO is strongly unstable. It means that a ray launched in the near vicinity of a $\mathrm{PO}$ will diverge in an exponential way. This instability is the origin of the extreme sensitivity to initial conditions that characterises chaotic systems. Due to the above correspondence, a typical mode may be considered as built upon a whole set of POs. Thus, in any surface element, one can derive a local expression of the transverse field $\psi_{s}(\mathbf{r})$, resulting from the superposition of a large number of plane waves of random directions and amplitudes, but with a fixed transverse wave number as shown by Berry in $[19,20]$ :

$$
\psi_{s}(\mathbf{r})=\sum_{n=0}^{N} b_{n} \exp \left(-i \kappa_{n} \cdot \mathbf{r}\right)+c . c
$$

where $b_{n}$ is the amplitude and $\boldsymbol{\kappa}_{n}$ the wave vector of the plane wave $n$. That is

$$
\psi_{s}(\mathbf{r})=\sum_{n=0}^{N} a_{n} \cos \left(\boldsymbol{\kappa}_{n} \cdot \mathbf{r}+\chi_{n}\right)
$$

with $\left\|\boldsymbol{\kappa}_{n}\right\|=\kappa$ and $\boldsymbol{\kappa}_{n}=\left(\kappa \cos \alpha_{n}, \kappa \sin \alpha_{n}\right)$, in which the angles $\alpha_{n}$ and $\chi_{n}$ are random variables. This expression defines a typical eigenmode of the chaotic structure, of energy $E=\frac{\kappa^{2}}{2}$ with $\kappa^{2}=\beta_{c o}^{2}-\beta_{n}^{2}$. Eq. (9) leads to a speckle-like pattern, as shown in Figure 3 : the intensity of a so-called speckle mode is statistically uniformly distributed over the whole billiard. The

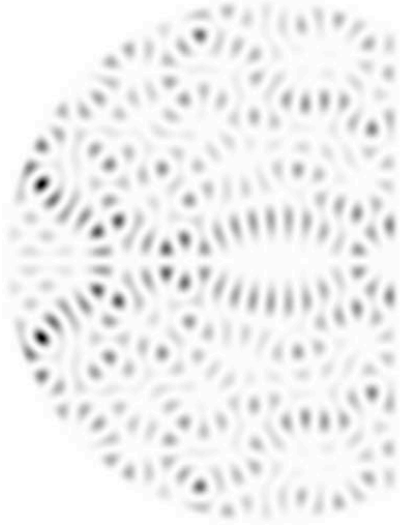

FIG. 3 A typical ergodic eigenmode (squared amplitude), in the D-shaped billiard.

mode presented in Figure 3 results from the Plane Wave Decomposition Method (PWDM) [21] that allows to calculate modes of a closed two-dimensional structure, with Dirichlet boundary conditions.

\subsubsection{Scar modes of a chaotic billiard}

The speckle-like behaviour of modes is generic in a chaotic billiard. However, by calculating all the modes of a chaotic billiard with the PWDM, some of them seem not to follow the generic behaviour described above. They are called scars because they present a high localisation of the intensity in the vicinity of some unstable POs of the billiard in the real space. Moreover, in the Fourier space, the directions of the POs are clearly emphasised. Some scar modes are shown in Figure 4. Numbers of explanations have been proposed, based on different approaches for this non-generic behaviour [20]-[22]. In this paper, we will not propose a theoretical analysis of the existence of scars, but rather focus on the 2-bounce PO and the scars that localise their intensities along it. The stability of a PO depends on the behaviour of the so-called monodromy matrix [23]. The latter is, in general, a chain of $2 \times 2$ matrices, alternatively associated to the propagation in straight lines between two reflections, and to the reflection itself at the boundary. This is analogous to the transfer matrix of optical elements, in optics. The eigenvalues of this matrix are related to the stability of the PO. In the case of our chaotic billiard, the largest eigenvalue takes the form $e^{\lambda \mathcal{L}_{q}}$ in which $\mathcal{L}_{q}$ is the length of the orbit $q$. For a given typical instability rate $\lambda$, the longer the orbit is, the more unstable it is. Thus the 2-bounce $\mathrm{PO}$ is one of the least unstable POs, and this turns out to be crucial in order to enhance the 2-bounce PO scars.
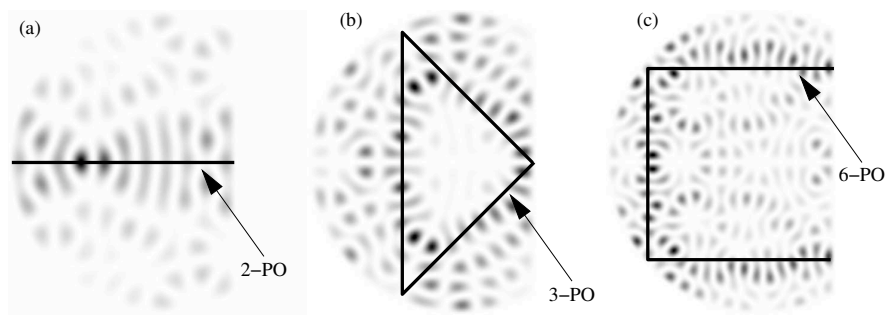

FIG. 4 Squared amplitudes of Scars of the D-shaped fibre, localising their intensities along the periodic orbits with 2, 3 and 6 bounces. 


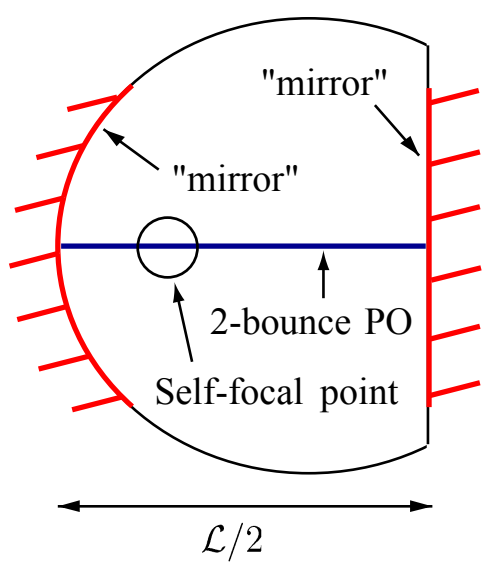

FIC. 5 Schematic representation of the transverse cross section of the D-shaped optical fibre as a Fabry-Perot resonator.

We now give some ideas to understand the presence of these scar modes by comparing the D-shaped billiard with an unstable Fabry-Perot resonator, made of a plane mirror and a concave mirror (Figure 5). From this analogy, the scars of the 2-bounce PO can be associated to the transverse modes of the resonator. Let $\mathcal{L}$ be the length of the 2-bounce PO. After a round trip through the lossless resonator, the cumulated phase is given by $\kappa \mathcal{L}-\Delta \phi-\pi / 2$ where $\Delta \phi$ is a phase shift due to the reflection on non-metallic boundaries, and $\pi / 2$ is a phase shift due to a particular point of the 2-bounce PO, the self-focal point. Rays launched from this point along the 2bounce PO, come back to focus on that point after 2 bounces. The condition of resonance reads

$$
\kappa \mathcal{L}-\Delta \phi-\pi / 2=2 \pi p
$$

where $p$ is an integer. From this condition of constructive interference on the transverse wave number $\kappa$, and for different values of the integer $p$, one can get the modes of the Dshaped billiard that correspond to this quantisation. It appears that these wave numbers correspond to the 2-bounce PO scar modes.

\section{AMPLIFICATION}

The spatial structure of scar modes and their regular repartition in the spectrum make them particularly interesting objects for some applications. From a fundamental point of view, the manipulation of these specific objects is also a real challenge. Thus, we strive to enhance these objects among all the modes. A single mode excitation is practically impossible in a highly multimode fibre [14]. Therefore, we propose to amplify this family of modes selectively. Indeed, their particular structure (spatial and phase space localisation of the intensity) in contrast with the other modes, makes these modes particularly suited for amplification. We propose a way of selecting a family of scar modes by a precise control of the amplification mechanism.

\subsection{Differential amplification}

A gain medium is introduced in the optical fibre in order to perform an amplification process. This technique is similar to
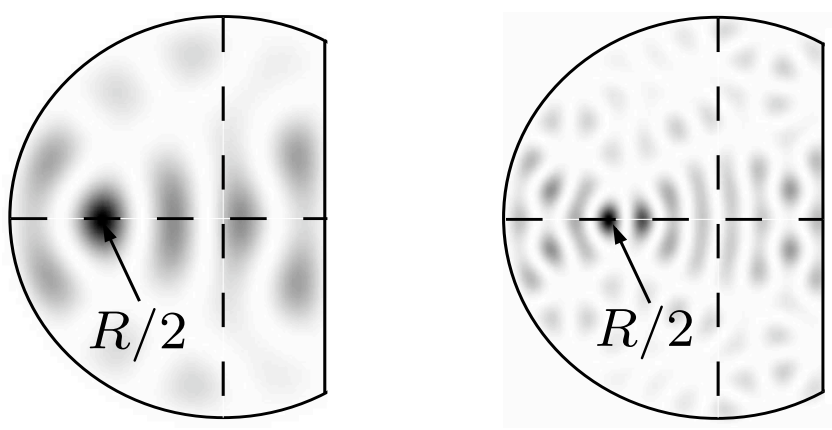

FIG. 6 Two scar modes : the maximum of intensity of these modes is localised on the same area, at the half-radius from the centre of the truncated circle (opposite side from the truncation)

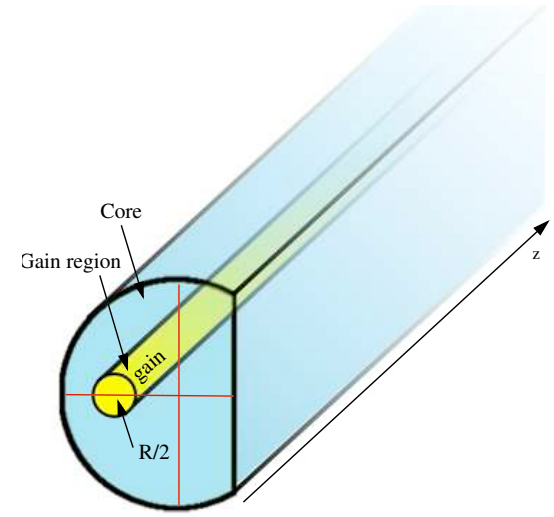

FIG. 7 The D-shaped optical fibre with gain localised on the maximum of intensity of scars, along the propagation length

the one commonly used in DCF amplifiers [10, 24], but has to be adapted in an unusual way. The main adaptation is that the signal to amplify should propagate in the whole D-shaped area, that is, for an amplifier, in the inner-cladding. Thus, the signal and the pump both propagate in the inner-cladding. In the following, we call the D-shaped fibre the core of the fibre, and the doped core the doped region. Indeed, it is worth noting that the difference between optical indices of the core and the doped region has to be low enough in order to avoid any guided mode in the latter. In the introduction, we mentioned the need of an optimised overlap between the pump intensity distribution and the doped region. The question is also relevant for the signal. To selectively amplify scar modes among all the excited modes, a differential amplification should be performed: (a) The scar modes we consider localise their intensities along the 2-bounce PO, suggesting to position the gain area along this direction. Then, in the semiclassical limit, a ray launched along the 2-bounce PO will pass through the gain medium more often than other trajectories. Thus, the modes that build on these trajectories might be amplified more efficiently than the others. This analysis is validated by inspecting the Husimi representation of the modes and will be published elsewhere. (b) On the other hand, the overlap between the gain region and the maximum of intensity of scars is a crucial point. This idea is based on recent studies made in the domain of random lasers. Information related to random lasers can be found in $[25,26]$ and we here briefly recall some important results for our purpose. Disordered media associated to random lasers present strongly localised modes. By introducing 
a gain medium on the maximum of intensity of these modes, a lasing effect is observed on the localised modes of the passive linear system. We try to apply this result to amplify scar modes. All scar modes of the 2-bounce PO present a maximum of intensity in the vicinity of $R / 2$, as shown in Figure 6. This position is not quite surprising, since it is the focal point of a concave mirror of radius $R$. Therefore, it seems natural to localise the gain medium by centring it around the focal point, as presented in Figure 7.

\subsection{Amplification scheme}

The fibre is doped with Ytterbium. The wavelengths for the pump and the signal are respectively $\lambda_{p}=980 \mathrm{~nm}$ and $\lambda_{s}=1064 \mathrm{~nm} .980 \mathrm{~nm}$ corresponds to the maximum of absorption of $\mathrm{Yb}$. The spectra of emission and absorption of $\mathrm{Yb}$ are presented in Figure 8. Usually, in order to facilitate Ytter-

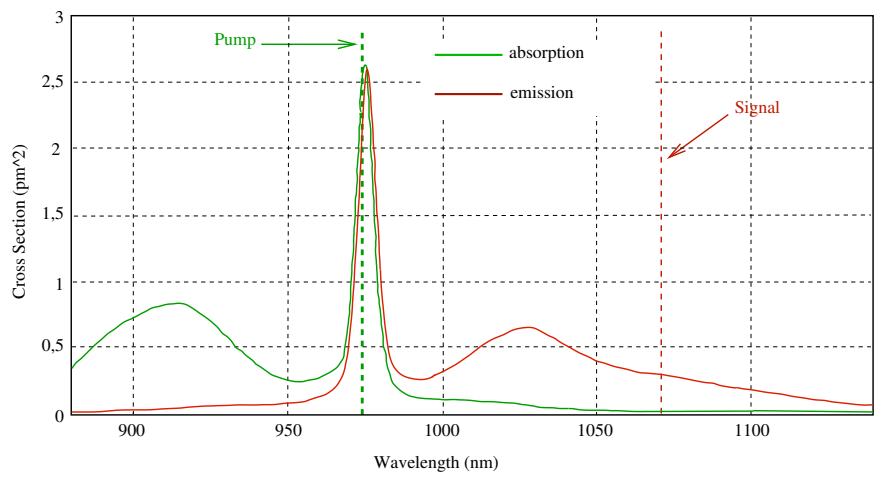

FIG. 8 Spectrum of absorption (green line) and emission (red line) of Ytterbium. The signal and pump wavelengths are specified.

bium incorporation in the optical fibre, Aluminium is also introduced. The effect of Aluminium is mainly to increase the optical index mismatch between the core and the doped area. In order to avoid any guided mode in the doped area, no Aluminium has been introduced in our optical fibre. One consequence is that the incorporation of $\mathrm{Yb}$ is less controlled. The optical index of the doped region is mainly determined by the concentration of $\mathrm{Yb}([\mathrm{Yb}])$ introduced. In our case, [Yb] has to be very low, in comparison to standard Ytterbium-dopedfibre-amplifiers, and is around $1000 \mathrm{ppm}$. The difference of optical indices is then around $\Delta n \simeq 5 \times 10^{-4}$. Numerical simulations showed that no guided mode can persist in this structure. Moreover, the refractive index changes due to the pump power $[10,27]$ involved in the fibre are negligeable, with respect to $\Delta n$.

Ytterbium is a four-level system, but the life-times of the levels that create non-radiative transitions are neglected here. Starting with this hypothesis, one can write the population evolution equations, that leads to the equation of evolution of the pump and signal intensities. Let $N_{1}\left(N_{2}\right)$ be the population density of the lower (upper) energy level

$$
\begin{aligned}
N_{t} & =N_{1}+N_{2} \\
\frac{d N_{1}}{d t} & =-\frac{\sigma_{p a} I_{p}}{h v_{p}} N_{1}+\frac{\sigma_{s a} I_{s}}{h v_{s}}\left(\eta_{s} N_{2}-N_{1}\right)+\frac{N_{2}}{t_{s p}}
\end{aligned}
$$

where $N_{t}$ is the total population density. $I_{p}$ is the pump intensity at $980 \mathrm{~nm}, I_{s}$ the signal intensity at $1064 \mathrm{~nm}, \sigma_{p a}$ (resp. $\sigma_{s a}$ ) the absorption cross section for the pump (resp. the signal), $v_{p}$ and $v_{s}$ are the frequencies associated to the transitions, $\eta_{s}=\frac{\sigma_{s e}}{\sigma_{s a}}$ with $\sigma_{s e}$ the emission cross section for the signal, and $t_{s p}$ the life-time of the upper level for the spontaneous emission. In the stationary state, Eq. (12) is

$$
\begin{aligned}
& N_{2}(x, y, z)=\frac{\frac{I_{p}}{I_{p 0}}+\frac{\frac{I_{s}}{I_{s 0}}}{1+\eta_{s}}}{1+\frac{I_{p}}{I_{p 0}}+\frac{I_{s}}{I_{s 0}}} N_{t} \\
& N_{1}(x, y, z)=\frac{1+\frac{\eta_{s} I_{s 0}}{1+\eta_{s}}}{1+\frac{I_{p}}{I_{p 0}}+\frac{I_{s}}{I_{s 0}}} N_{t}
\end{aligned}
$$

where $I_{p 0}\left(I_{s 0}\right)$ is the pump (signal) intensity of saturation. The variation of intensities of the pump and signal are deduced from the expression of the energies involved in the absorption and stimulated emission processes. The energy absorbed by units of time and surface along $d z$ is

$$
\begin{aligned}
-d I_{p} & =\sigma_{p a} I_{p} N_{1} d z \\
-d I_{s} & =\sigma_{s a} I_{s}\left(N_{1}-\eta_{s} N_{2}\right) d z
\end{aligned}
$$

Thus, one can write

$$
\begin{aligned}
& \frac{d I_{p}}{d z}=-\sigma_{p a} N_{1} I_{p}(z) \\
& \frac{d I_{s}}{d z}=\sigma_{s a}\left(\eta_{s} N_{2}-N_{1}\right) I_{s}(z)
\end{aligned}
$$

describing the energy transfer process between the pump and the signal. For more details, please refer to [28].

\subsection{Numerical model of the amplification}

In this part, we describe the model of the amplification process, used in the numerical simulations presented below. In Eqs. (17), the coefficients of absorption $\alpha_{a b s}=-\sigma_{p a} N_{1}(z)$ and amplification $\alpha_{a m p}=\sigma_{s a}\left(\eta_{s} N_{2}(z)-N_{1}(z)\right)$ depend on the intensities $I_{p}(z)$ and $I_{s}(z)$. In the numerical simulations, the length of propagation of both the pump and the signal is discretised in steps of length $\Delta z=5 \times 10^{-6} \mathrm{~m}$. The evolution of the pump and signal amplitudes is calculated through the "Beam propagation method" $[29,30]$. At each step $\Delta z$, we account for the amplification (resp. depletion) of the signal (resp. the pump) by multiplying their amplitudes by $e^{\alpha_{a m p} \Delta z / 2}$ (resp. $\left.e^{\alpha_{a b s} \Delta z / 2}\right)$.

\section{RESULTS}

In all the numerical simulations we performed, the parameters used are based on the physical characteristics of the actual optical fibre. The numerical initial condition used for the pump evolution corresponds to an illumination by a focussed laser beam, ensuring an optimised coupling with a large number of speckle modes. The input powers are $6 \mathrm{~W}$ for the pump, and $100 \mu \mathrm{W}$ for the signal. The cross sections for the pump and signal wavelengths have the following values : $\sigma_{p a}=2.65 \times 10^{-24} \mathrm{~m}^{2}, \sigma_{s a}=5.56 \times 10^{-26} \mathrm{~m}^{2}$ and $\sigma_{s e}=6.00 \times 10^{-25} \mathrm{~m}^{2}$. The life-time of the upper level for spontaneous emission is $t_{s p}=10^{-3} \mathrm{~s}$. The concentration of Ytterbium given before implies $N_{t}=10^{26} \mathrm{~m}^{-3}$. 
To analyse the guided modes present in the propagating signal we calculate what we call the pseudo-time-frequency spectrum $C(\kappa ; z)$ in which $z$ is the pseudo-time [31]. More precisely, $C(\kappa ; z)$ is obtained by the Fourier transform of the correlation function of the propagating field $\psi_{s}(\mathbf{r}, z)$ with its initial condition, along a propagation length $L_{z}$

$$
\begin{aligned}
C(\kappa ; z) & =\int_{z-\left(L_{z} / 2\right)}^{z+\left(L_{z} / 2\right)} d z^{\prime}\left[\iint d \mathbf{r} \psi_{s}^{*}(\mathbf{r}, 0) \psi_{s}\left(\mathbf{r}, z^{\prime}\right)\right] e^{-i \beta(\kappa) z^{\prime}} \\
& \simeq \sum_{n} A_{n}(z) \delta_{\epsilon}\left(\kappa_{n}-\kappa\right)
\end{aligned}
$$

in which $C(\kappa ; z)$ is clearly written in a form that exhibits peaks for transverse wave numbers associated to the eigenmodes of the fibre. We present the results for a fibre length $(\simeq 50 \mathrm{~m})$ for which the maximum of amplification is achieved. Let us consider a spatially incoherent initial condition for the signal, resulting from a random superposition of a large number of plane waves. The wave numbers are randomly distributed from 0 to $\tilde{\kappa}=\kappa_{\max } / 4$ (where $\kappa_{\max }=\beta_{c o}^{2}-\beta_{c l}^{2}$ ) and the corresponding initial spectrum is almost uniform over this range of wave numbers. The narrow selection of the direction in the
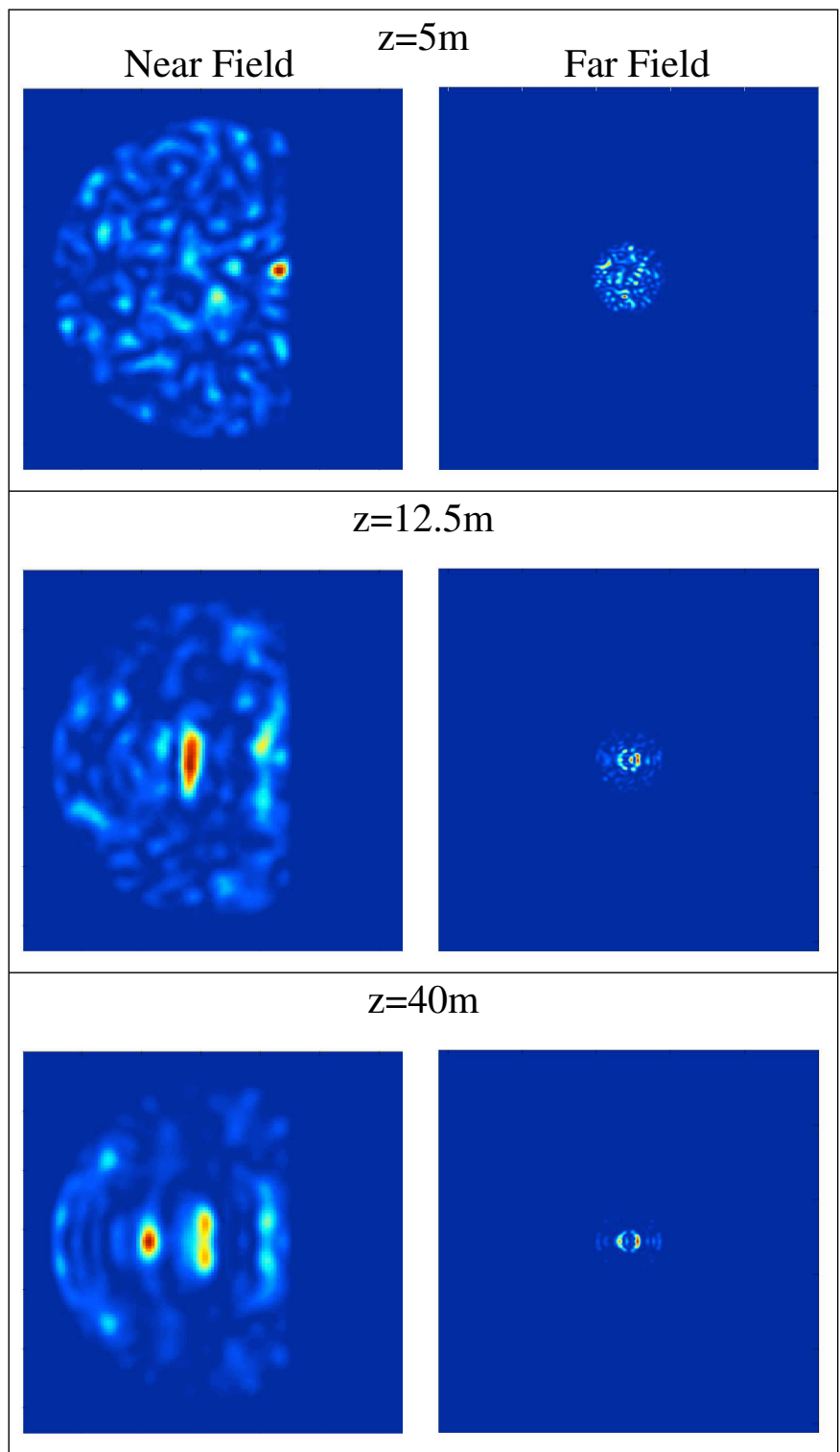

FIC. 9 Near field and far field for 3 different lengths of the fibre. The selection of the 2-bounce PO is noticeable, as well in the near field, as in the far field pictures. vicinity of the $\mathrm{PO}$ is obvious in the spatial distribution of the signal field (Figure 9).

Of course, the near field does not show a single scar mode, as the initial condition includes a dozen of them, but the localisation of the light along the 2-bounce PO is clearly demonstrated. Moreover, the selection of the direction becomes obvious if one looks at the far field, as shown in Figure 9.

In Figure 10(a), the evolution of $C(\kappa ; z)$ along the propagation $z$ is computed with $L_{z}=1.3 \mathrm{~m}$ and with $z$ varying by steps of $0.65 \mathrm{~m}$ for $z=0$ to $50 \mathrm{~m}$. Figure 10(a) represents the average spectrum for 10 different inputs. There is a drastic mode selection along the propagation. Each noticeable peak corresponds to the transverse wave number of a scar mode. Almost all scar modes are significantly amplified up to $p=12\left(\kappa_{12} \simeq \tilde{\kappa}\right)$ but not with the same efficiency. Figure 10(b) represents the evolution along $z$ of the intensity associated to the wave number of each scar mode in a semi-logarithmic scale. The intensity evolutions along $z$ follow an exponential law, until the gain saturation is achieved. This exponential growth is explained by considering that the spatial distribution of the intensity of one mode increases uniformly along the propagation. The amplification rate of each scar mode can be directly deduced from the slope of the exponential growth in the semi-log representation. It becomes obvious that some scars as the scars of order 1,3,4 and 6 have a higher amplification rate than the scars of order 2 or 5 . This differential amplification can be related to the overlap between the scar modes spatial intensity distributions and the doped region. This is illustrated in Figure 11, where the overlap integral between the doped region and the intensity of the 2-bounce PO scar modes has been plotted. The good agreement between the amplification rates and

(a)

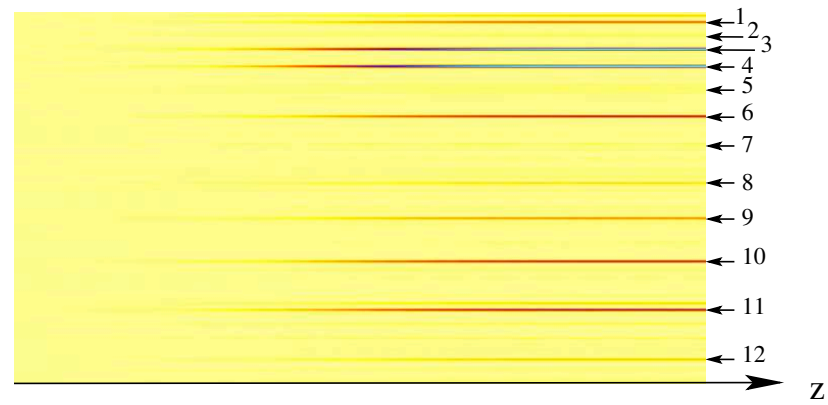

(b)

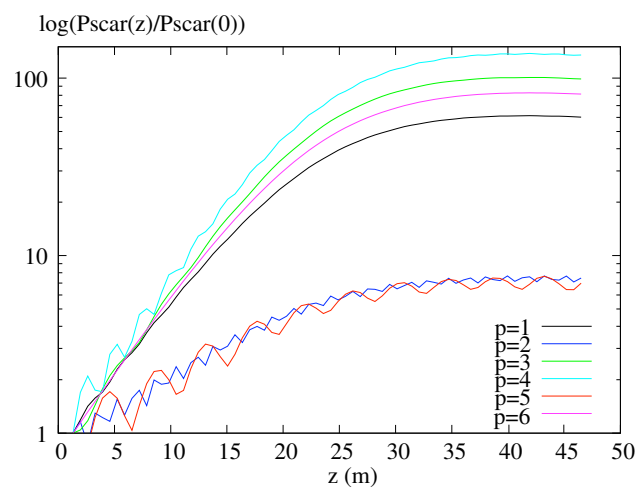

FIC. 10 (a) 2D-Evolution of $C(\kappa ; z)$. Each peak corresponds to a scar mode, denoted here by their order $p$. (b) Evolution of the intensities of scar modes along the propagation $z$. 


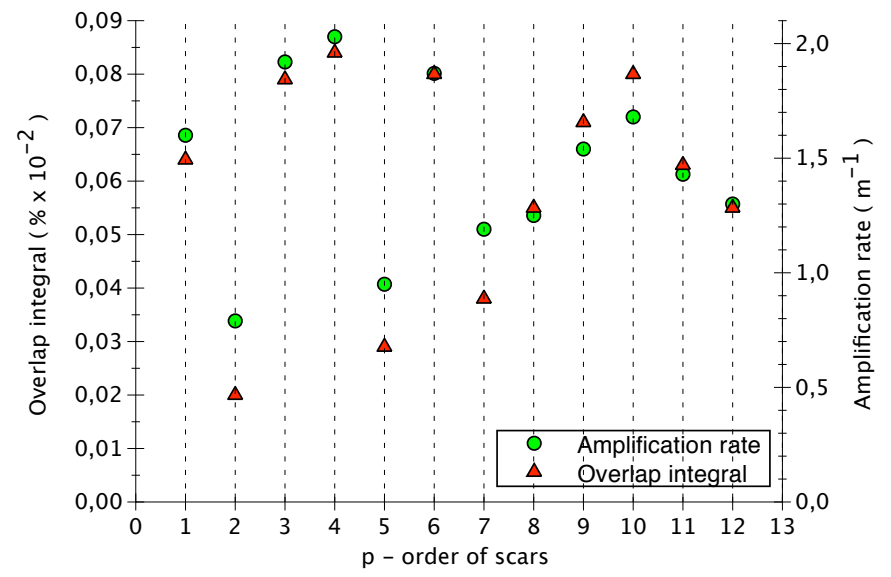

FIG. 11 Qualitative comparison of the overlap integral between the intensity of the calculated scar modes and the doped area, and the amplification rates measured in Figure 10(b).

the overlap integrals is clearly seen : scars of order $p=1,3,4,6$ (and 10) have a good overlap with the gain region, and thus have a higher amplification coefficient, whereas scars of order $p=2,5$ (and 7) have a poor overlap, and are not efficiently amplified.

\section{CONCLUSION AND PROSPECTS}

The efficiency of the process of amplification of scar modes has been numerically demonstrated, and we have analysed the differential amplification of the modes with respect to their overlap with the position of the gain medium. All the parameters used in the numerical simulations are taken from the actual optical fibre, and the experiment is under progress. We briefly present the experimental scheme (Figure 12). A YAGlaser@ 1064 nm (350 mW) and a laser diode @ 980 nm (10 W) are used, respectively for the signal and the pump. The signal is expanded and filtered, in order to optimise the illumination of the fibre input. The output of the laser diode is collimated and then focussed at the D-shaped fibre input, in order to distribute the intensity over all the modes of the fibre. The fibre length is $50 \mathrm{~m}$, and is wrapped around a $30 \mathrm{~cm}$ diameter spool. At the fibre output, a dichroic mirror separates the two wavelengths. Different analyses can be performed as, for instance,

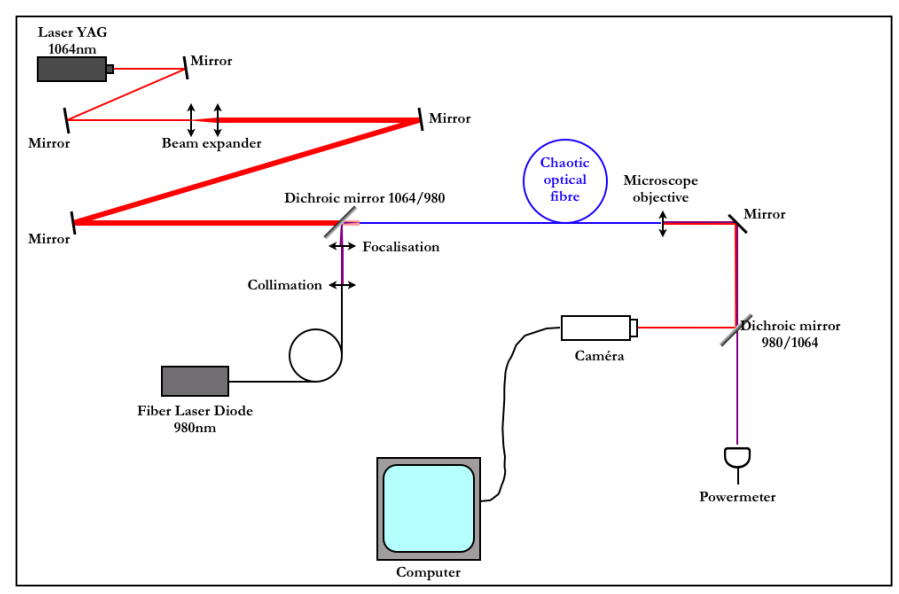

FIG. 12 A scheme of the experiment that is under progress. the visualisation of the near and far fields, or a spectrum analysis.

\section{ACKNOWLEDGEMENTS}

We gratefully acknowledge P. Aschiéri and W. Blanc for fruitful discussions, and also M. Udé and S. Trzesien for providing physical data concerning the D-shaped fibre amplifier. This work is supported by ANR contract No. 05-JCJC-0099-01.

\section{References}

[1] H.-J. Stöckmann, Quantum chaos : an introduction (Cambridge University Press, Cambridge, England, 1994).

[2] R. Chang and A. Campillo, "Optical processes in microcavities" World Scientific, (1996).

[3] W. Fang, A. Yamilov, and H. Cao, "Analysis of high-quality modes in open chaotic cavities" Phys. Rev. A 72, 023815 (2005).

[4] S. Sunada, T. Harayama, and K. Ikeda, "Multimode lasing in twodimensionan fully chaotic cavity lasers" Phys. Rev. E 71, 046209 (2005).

[5] M.Lebental, J. Lauret, R. Hierle, and J. Zyss, "Highly directional stadium-shaped polymer microlasers" Appl. Phys. Lett. 88, 031108 (2006).

[6] A. Savchenkov, A. Matsko, D. Strekalov, V. Ilchenko, and L. Maleki, "Enhancement of photorefraction in whispering gallery mode resonators" Phys. Rev. B 74, 245119 (2006).

[7] J. Nockel and A. Stone, "Ray and waves chaos in asymetric resonant optical cavities" Nature 385, 45 (1997).

[8] C. Gmachl, F. Capasso, E. Narimanov, J. Nockel, A. Stone, J. Faist, D. Silco, and A. Cho, "High-Power Directional Emission from Microlasers with Chaotic Resonators" Science 280, 1556 (1998).

[9] T. Haryama, T. Fukushima, P. Davis, P. Vaccaro, T. Miyasaka, T. Nishimura, and T. Aida, "Lasing on scar modes in fully chaotic microcavities" Phys. Rev. E 67, 015207 (R) (2003).

[10] E. Desurvire, Erbium doped fiber amplifiers (Wiley Interscience, 1994).

[11] P. Leproux, S. Fevrier, V. Doya, P. Roy, and D. Pagnoux, "Modeling and Optimization of Double-Clad Fiber Amplifiers Using Chaotic Propagation of the Pump" Opt. Fiber. Technol. 7, 324 (2001).

[12] V. Doya, 0. Legrand, F. Mortessagne, and C. Miniatura, "Speckle statistics in a Cahotic Multimode Fiber" Phys. Rev. E 65, 056223 (2002).

[13] V. Doya, 0. Legrand, and F. Mortessagne, "Optimized absorption in a chaotic double-clad fiber amplifier" Opt. Lett. 26, 872 (2001).

[14] V. Doya, 0. Legrand, and F. Mortessagne, "Light Scarring in an optical fiber" Phys. Rev. Lett. 88, 014102 (2002).

[15] A. Snyder and D. Love, Optical Waveguide Theory (Chapman and Hall, 1983).

[16] A. D. Stone, "Einstein's unknown insight and the problem of quantizing chaos" Phys. Today 8, 37-43 (2005).

[17] M. Gutzwiller, "Periodic orbits and quantum quantization conditions" J. Math. Phys. 12, 343 (1971).

[18] M. Gutzwiller, Chaos in classical and quantum mechanics (Springer-Verlag, New York, 1990). 
[19] M. Berry, "Regular and irregular semiclassical wavefunctions" J. Phys. A - Math. Gen 10, 2083-2091 (1977).

[20] M. Berry, "Semiclassical mechanics of regular and irregular motion" in Session XXXVI - Chaotic behaviour of deterministic systems (Les Houches, France, 1981).

[21] E. Heller, "Wave packet dynamics and quantum chaology" in Session LII - Chaos and quantum physics (Les Houches, France, 1989).

[22] E. Bogomolny, "Smooth wavefunctions of chaotic quantum systems" Physica D 31, 169-189 (1988).

[23] B. E. A. Saleh and M. C. Teich, Fundamentals of photonics (John Wiley and Sons, 1991).

[24] H. Po, "High gain power amplifier tandem pumped by GaAlAs phased array" in Proc. Conf. Opt. Fibre Commun. PD7 (1989).

[25] P. Sebbah and C. Vanneste, "Random lasers in the localized regime" Phys. Rev. B 66, 144202 (2002).
[26] C. Vanneste and P. Sebbah, "Selective excitation of localized modes in active random media" Phys. Rev. Lett. 87, 183903 (2001).

[27] H. Garcia, A. Johnson, F. Oguama, and S. Trivedi, "Pump-induced nonlinear refractive-index in erbium- and ytterbium-doped fibers : theory and experiments" Opt. Lett. 30, 1261 (2005).

[28] A. Ghatak and K. Thyagarajan, Introduction to fiber optics (Cambridge University Press, 1999).

[29] M. Feit and J. Fleck, "Computation of mode properties in optical fiber waveguides by a propagating beam method" Appl. Opt. 17, 3990 (1978).

[30] V. Doya, 0. Legrand, and F. Mortessagne, "Optimized absorption in a chaotic double-clad fiber" Opt. Lett. 2, 872 (2001).

[31] C. Michel, V. Doya, 0. Legrand, and F. Mortessagne, "Selective amplification of scars in a chaotic optical fiber" Phys. Rev. Lett. 99, 224101 (2007). 